\title{
Effects of a Rhythmic-Play Exercise Program on Coordination in Preschool Children
}

\author{
Eri Yoshimi' ${ }^{1,2}$, Teruo Nomura1, Noriyuki Kida1 \\ ${ }^{1}$ Graduate School of Science and Technology, Kyoto, Japan \\ ${ }^{2}$ Tokiwakai College Early Childhood Education, Osaka, Japan \\ Email: e-yoshimi@tokiwakai.ac.jp
}

How to cite this paper: Yoshimi, E., Nomura, T., \& Kida, N. (2021). Effects of a Rhythmic-Play Exercise Program on Coordination in Preschool Children. Advances in Physical Education, 11, 207-220. https://doi.org/10.4236/ape.2021.112016

Received: February 23, 2021

Accepted: May 7, 2021

Published: May 10, 2021

Copyright $\odot 2021$ by author(s) and Scientific Research Publishing Inc. This work is licensed under the Creative Commons Attribution-NonCommercial International License (CC BY-NC 4.0). http://creativecommons.org/licenses/by-nc/4.0/

\section{(c) (i) (8) Open Access}

\begin{abstract}
This study was intended to clarify the effects of an exercise program emphasizing rhythmic play on different aspects of motor coordination in preschool children. Eighty preschoolers (46 four-year-olds, 34 five-year-olds) participated in the program for four weeks. Different components of coordination were evaluated before and after the intervention using the Coordination Field Test (CFT), a test battery developed by the Expert Committee on Coordination at Japan's Research Center in Physical Education. Two-factor repeated-measures ANOVA detected no significant interactions between measurement time and preschool year. Preschool year (age) had significant main effects on children's performance of a jump-over-crawl-under task $(F=$ 27.45), a zigzag-run task $(\mathrm{F}=10.03)$, and a side-stepping task $(\mathrm{F}=15.97)$; measurement time had significant main effects only in the last two $(\mathrm{F}=18.83$, 158.84, respectively). The global improvements observed on the zigzag-run and side-stepping tasks reveal that this rhythmic-play exercise program influences coordination skills related to agility. Conversely, children start displaying a need for opportunities to acquire (fine-motor) coordination skills related to dexterity around the age of four. Altogether, our findings confirm that the rhythmic-play exercise program examined here can effectively improve different aspects of coordination in preschool children.
\end{abstract}

\section{Keywords}

Coordination, Rhythmic Play, Exercise Program, Preschool Children

\section{Introduction}

\subsection{Coordination in Preschoolers Is Limited by Underdeveloped Motor Patterns}

Underdevelopment of basic motor patterns and skills in early childhood can lead 
to significant injuries (SCJ, 2017). This underdevelopment can be viewed as a consequence of immaturity in the nervous functions that govern muscle activity and movement by extension: this control is commonly known as coordination (Ikai, 1967). Decades ago, researchers found that coordination-related abilities are most easily developed between the ages of 4 to 6 (Ishikawa et al., 1987). Nonetheless, more recently, others found that 5- to 6-year-old children tested in 2007 had similar motor acquisition levels to 3- to 4-year-old children tested in 1985 (Nakamura et al., 2011). Delayed coordination skills, which normally develop rapidly starting at age 4 or 5 , account for motor immaturity in children in the modern era. Numerous field studies on this topic have been conducted in childcare settings: in one, Yoshida and Iwasaki (2014) found that kindergarten teachers were dissatisfied with how they carried out exercise instruction, feeling their approaches which were in need of improvement. Japan's Ministry of Education, Culture, Sports, Science and Technology (MEXT) (2017) revised its National Curriculum Standards for Kindergartens, adding new provisions about dealing with content related to health: "Children should become able to coordinate body movements through various exercises." Revised based on the Ministry's Exercise Guidelines for Early Childhood plan, this declaration presents the issue as a duty of educational institutions in the country. Exercise in childcare settings should help young children improve their motor skills and foster coordination through play and games in which they move their bodies. Such problems with exercise guidance and developmental delays in coordination affect preschool children in the modern era.

In Japan, the term "coordination" (chōsei-ryoku) was used in the 1968 revision of the National Curriculum Standards for Elementary Schools, stipulating "cultivating coordination by having children engage in various kinds of exercise suitably" among the goals for each school year. Coordination was also included as an element of physical fitness in the physical education guidelines of the 1969 MEXT National Curriculum Standards for Elementary Schools, which divide it into three exercise components: balance, dexterity (i.e., fine-motor skills), and agility. Matsuura (1998) called it "the ability to realize a single, cohesive, global or local movement by integrating the motion of different body parts and organs involved in locomotion." Further, Matsuura showed that evaluating coordination in terms of the degree to which such movements are correctly realized was a pragmatic way to deduce mental phenomena, for instance, those above-mentioned "psychological factors." These findings support coordination's classification as an element of physical fitness involved in controlling movement and motor skill that includes psychological elements. Maehashi (2017) explains the functional aspects of coordination in terms of four component abilities that allow individuals to execute movements accurately, smoothly, and effectively: dexterity: facility and skillfulness in utilizing one's body towards a goal; agility: swiftness in moving and responding with one's body; balance: remaining stationary or maintaining posture during body movements; and coordinability (kyōō-seî): an essential ability for complex motor learning, whereby the movements of two or more 
body parts are integrated into a single unified motion.

The above-mentioned Expert Committee on Coordination finalized its Coordination Field Test (CFT) format, a field test developed to investigate coordination, in 1981. Coordination is evaluated by this tool using three tasks/activities, which correspond to different component skills: jump-over-crawl-under (dexterity), side-stepping (agility), and zigzag-run (agility). The CFT can be used to quantify and evaluate coordination even in young children: its scoring and integrated rating criteria have been published.

Physical education research has shown that coordination training exercises improve coordination in preschool children. Numerous practical studies of exercise programs have explored the effectiveness of coordination training in educational settings (e.g., Hirai \& Kasahara, 2016; Kamimaru, 2010; Ueta et al., 2006; Yasumitsu \& Nogawa, 2010). Notably, age-appropriate coordination training has been reported to improve coordination in daycare attendees (Kozuka, Kuga, \& Watanabe, 2010), raising hopes that doing targeted exercises should be somewhat effective at increasing the coordination skills of preschoolers and juveniles. Unfortunately, few such studies have targeted preschool-age children to date.

The practical research literature includes numerous interventional studies of exercise programs designed for children that incorporate rhythmic dancing and movements (Evridiki, Aggeliki, \& Vassiliki, 2004; Takenawa, Takeda, Kikuchi, \& Nakagawa, 2008; Tomita, Otobuchi, \& Matsushima, 2014). Notably, Takahara, Sunami, and Taki (2015) ran a play exercise intervention consisting of five types of activities, jumping, throwing, upper-body strength (e.g., pole climbing, monkey bars), group activities involving the entire body (e.g., flag tag), and pair play with parents at home, to explore how motor abilities could be affected by physical play in young children. They found that their program led to significant improvements in children's fitness and motor skills. Similar effects have been demonstrated in previous research on exercise programs involving rhythmic play and other physically expressive activities. Furuichi (1995) contends that synchronizing and keeping in time with the same rhythm as other children produces psychological and physical pleasure, fostering mental development by encouraging them to spontaneously and voluntarily move together with the group. She showed that coordinating motion with rhythm involves physical expressivity, which brings about feelings of psychological and physical satisfaction. Furthermore, the first health-related aim in the National Curriculum Standard for Kindergartens, "to act positively and freely and to experience a sense of fulfillment" (MEXT, 2017), could be interpreted as designed to satisfy the needs of young children by providing them with opportunities to express themselves openly and creatively. Indeed, rhythmic play is well-suited to exercise programs targeting coordination, given the ability's intrinsic nature and overlap with psychological factors, meaning rhythmic activities that fulfill young children's needs could likewise influence their coordination skills. Therefore, it stands to reason that an exercise program intervention that incorporates rhythmic play could 
improve coordination skills in preschool children and even satisfy their needs in a way befitting early childhood development.

\subsection{Problem and Objective}

Research to date conducted by the authors has demonstrated that exercise programs that emphasize rhythmic play can improve motor skills in preschool-age children. It seems reasonable to predict that such interventions would also enhance coordination in this population, based on evidence in past work on the topic, including the authors' research history (Yoshimi \& Katayama, 2019; Yoshimi, 2020a, 2020b; Yoshimi, Nomura, \& Kida, 2021). However, few interventional studies of exercise programs focused on rhythmic play have utilized physical performance tests to evaluate coordination skills in childcare settings. Given this gap in the literature, trialing a rhythmic play-based exercise program in a group of preschool children, and then substantiating its effects on their coordination using relevant physical performance tests, seemed like a worthwhile endeavor.

In this study, the CFT was utilized to determine how such programs can influence coordination skills. These effects were evaluated in terms of an integrated rating calculated by combining an individual's performances on each of the three component tasks according to the test's scoring criteria. The raw performance was also examined separately for each task to gain insight into current coordination levels and our program's effectiveness in this young population.

This paper details the findings of a quasi-experimental study in which preschool children participated in a coordination exercise program that emphasizes rhythmic play. Children's pre- and post-intervention performances on the CFT were compared to determine the program's effectiveness and its effects on coordination in this age group.

\section{Method}

\subsection{Study Participants}

A total of 80 children attending a private kindergarten in Japan participated in the present study, comprising 46 members of a class for 4 -year-olds (M/F ratio: 24/22, mean age [T0]: $62.7 \pm 3.3$ months) and 34 members of a class for 5 -year-olds (18/16, $73.5 \pm 3.2$ months). (Below, these groups are referred to as "4-year-olds" and "5-year-olds," respectively, for simplicity.) Ideally, some of the children would have been randomly assigned to a control group. However, given the burden this would have placed on their parents and the time constraints posed by various events during the school year, it was decided to adopt a quasi-experimental (pre-post-intervention) design. Kindergarten facilities included a playground yard and day nurseries. Ethical considerations were fulfilled by explaining the study purpose in advance to the kindergarten principal, teachers, and children's parents/guardians, and enrolling only children whose parents had consented to their participation. The Research Ethics Committee of the Kyoto 
Institute of Technology approved this study (approval no. 2019-73).

\subsection{Procedure}

The study timeline consisted of a four-week non-exercise-intervention period followed by a four-week exercise intervention period (Figure 1). The same subjects were followed for the entire study period. During the non-exercise-intervention period, children did not follow any specific exercise program but participated in classroom activities normally. During the exercise intervention period, they performed a brief, six-minute exercise program that incorporated coordination exercises, primarily involving rhythmic play, once per day, for 20 sessions in total. Subjects were evaluated at three-time points to ascertain whether this intervention resulted in changes in their coordination abilities. That is, before the non-exercise-intervention period (T0; "1st test"), between the non-exercise-intervention and exercise intervention periods (T1; "pre-test"), and after the end of the exercise intervention period (T2; "post-test"). This protocol was designed based on previous work (Umezaki, Nakatani, Yamamoto, Nakasuka, \& Hashimoto, 2013). The study was conducted from December 2019 to February 2020.

\subsection{Exercise Program}

The exercise program was the same as described in previous work by the authors (Yoshimi et al., 2021). The exercise program consisted of six events: walking, jumping, rotation, support, rhythm, and balance. The movements (which included the basic movements shown in "movement to balance the body") included standing, sitting, and rotating. In the "movement to move the body," walking, running, hopping, and jumping were incorpo-rated, as shown under "diversification of movement" in the early childhood-movement guidelines. The content was designed to ensure that the seven kinds of coordination skills, orientation, adaptation, rhythm, reaction, balance, coupling, and differentiation, could be learned equally well. The tempo of the music used in the exercise programs was allegretto. Children performed each activity's motions according to their ability, progressing to successive steps as they became more proficient to ensure their motivation and interest would remain high and avoid boredom with the activities. Each activity's instructions and sequence of movements were explained to parents in advance, with demonstrations. Children did the program in a location where they had plenty of space to move their bodies: the schoolyard or

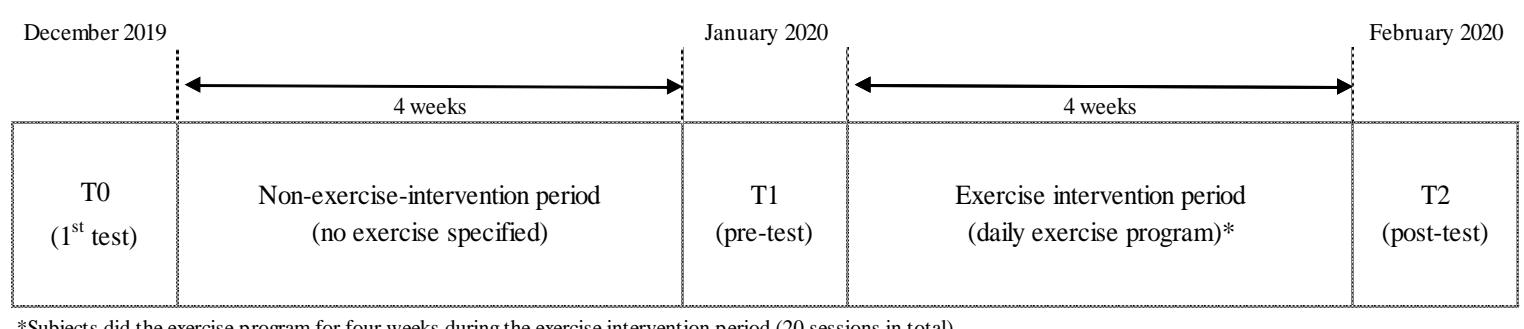

*Subjects did the exercise program for four weeks during the exercise intervention period (20 sessions in total)

Figure 1. Measurement protocol. 
a playroom, depending on their daily rhythms, weather, school events, and other factors.

\subsection{Coordination Evaluation}

\section{Coordination Field Test}

This study adopted the CFT, a battery of tasks developed at the RCPE mentioned above (Kurimoto et al., 1981). The CFT consists of a jump-over-crawl-under task, a zig-zag run task, and a side-stepping task. In the first two, better coordination is reflected by shorter completion times; in the third, it is signified by higher numbers of repetitions. Children performed each task twice, one practice run, followed by one real trial (for which performance was logged), to ensure their peers would not become unduly fatigued during the school day while waiting to be tested. However, failed attempts were repeated until a valid measurement was obtained.

\subsection{Statistical Analysis}

Records of children's performance on each CFT task at the three-time points above were compared using two-factor repeated-measures ANOVA to check for interactions between measurement time factors (T0, T1, T2) and preschool year (i.e., 4-year-olds, 5-year-olds). Significance level was adjusted for multiple comparisons using the Bonferroni correction if a significant main effect of measurement time was observed. IBM SPSS Statistics V25.0 software was used for all statistical analyses. Significance was set at $p<0.05$.

\section{Results}

\subsection{Physical Changes and Task Performance}

Kindergarten staff measured subjects' height and weight one month apart, once in January and once in February, to estimate (one-month) changes in physical characteristics in parallel with the intervention. Subjects increased in height and weight over the exercise intervention period, with 4 -year-olds growing by 0.31 $\mathrm{cm} / 0.1 \mathrm{~kg}$ and 5 -year-olds by $1.1 \mathrm{~cm} / 0.21 \mathrm{~kg}$. The program's effectiveness was assessed in terms of how much children's performance on each CFT task improved after with respect to before the exercise intervention period (i.e., T2 T1). The 4-year-old group showed improvements on all CFT components, completing the jump-over-crawl-under task $1.93 \mathrm{~s}$ quicker, the zigzag-run task $1.56 \mathrm{~s}$ quicker, and the side-stepping task with 6.54 more reps on average at $\mathrm{T} 2$ than T1. The 5-year-old group also showed improvements on the zigzag-run and side-stepping tasks, by $0.88 \mathrm{~s}$ and 7.88 reps, respectively; however, they performed slightly worse on the jump-over-crawl-under task after the intervention, completing it $0.62 \mathrm{~s}$ slower.

\subsection{Integrated Coordination Ratings}

Coordination was also evaluated holistically using an integrated CFT score, 
measured in points, and the rating instructions established by the test's developers (RCPE, 1981). Overall coordination was rated using the provided five-step scale developed by the Expert Committee on Coordination based on the points totals for the three tasks: Excellent (A), Superior (B), Normal (C), Poor (D), and Unusually Poor (E). Children in the 4-year-old group were found to have better overall coordination-as measured by the integrated CFT score-after than before the exercise program. Boys' and girls' (mean) scores at T0 and T1 were rated $\mathbf{D}$, signifying poor coordination; by $\mathrm{T} 2$, their scores had improved to $\mathbf{B}$ ratings, signifying superior coordination (boys = T0: 10.1, T1: 11.7, T2: 17.3; girls = T0: 10.7, T1: 13.1, T2: 17.7). Similarly, 5-year-old children were found to have better overall coordination after than before the program. At T0, boys received an integrated $\mathrm{E}$ rating, signifying unusually poor coordination, while girls received an integrated $\mathbf{D}$ rating, signifying poor coordination. By $\mathrm{T} 2$, both sexes' scores had improved to $\mathrm{C}$ ratings, signifying normal coordination (boys = T0: 9.8, T1: 11.5, T2: 17.6; girls = T0: 9.3, T1: 11.3, T2: 16.1).

\subsection{Performance Changes and the Effects of the Preschool Year}

No significant interactions between measurement time and preschool year were detected by two-factor ANOVA (Table 1). Preschool year had the main effect on jump-over-crawl-under performance $(\mathrm{F}=27.45, p=0.00)$; however, the measurement time did not reach statistical significance. Both factors had significant main effects on zig-zag-run performance (time: $\mathrm{F}=18.83, p=0.00$; year: $\mathrm{F}=$ $10.03, p=0.02$ ) and side-stepping performance (time: $\mathrm{F}=158.84, p=0.00$; year: $\mathrm{F}=15.97, p=0.00)$.

\section{Discussion}

\subsection{Physical Characteristics and Integrated Ratings}

Our preschoolers' one-month changes in body height and weight were similar to

Table 1. Two-factor ANOVA (measurement time $\times$ preschool year) for each component of the Coordination Field Test.




values reported for the same age group in the National Growth Survey on Preschool Children (Ministry of Health, Labour and Welfare, 2010). Early childhood is a time of significant development, not only in terms of physical attributes but also of the nervous system. Considering the brief length of our four-week exercise program, the changes observed in subjects' task performances were probably more closely related to physical fitness changes (i.e., motor skills) than changes in physique or stature.

Both 4- and 5-year-old preschoolers exhibited higher overall coordination-in terms of integrated CFT rating-after our intervention than before it. The CFT was similarly used in a previous work by Yanagisawa (1994): in comparison, nearly all participants-except some male children-earned a B (superior) rating, denoting a study population with good coordination skills overall. Thus, while our participants seem to have had more deficient motor coordination than populations examined in previous work and reference values for integrated CFT rating, the fact that their ratings improved after the intervention seems to indicate that our exercise program influenced their coordination.

\subsection{Intervention Effects on Coordination Abilities}

Children's performance on the jump-over-crawl-under task was not significantly improved after the intervention. For 5-year-olds, the trendline was almost flat; for 4-year-olds, the completion time was slightly lower after the intervention but not significantly so. Based on these observations, we conclude that our program had only a slight effect on dexterity in 4-year-olds and no impact on dexterity in 5 -year-olds. This small disparity in performance echoes a similar tendency observed in a recent assessment of different jumping tasks. Katoh and Kobayashi (2020) evaluated the degree to which subjects had acquired these jumping movements utilizing an aggregate score. Both 4- and 5-year-old children were more proficient at jumping than their 3-year-old peers, but the difference between 4- and 5-year-olds' scores was not significant, suggesting that children's performance of jumping-related movements does not improve much after four years of age. Perhaps a trade-off between quality and quantity was responsible for our subjects' lack of improvement on this task. This speculation is supported by the findings of Tanaka et al. (2017), who evaluated both fundamental motor skills and motor performance in preschool children, corresponding to movement quality and quantity, respectively. Subjects who completed the jump-overcrawl-under task more accurately-specifically, clearing the horizontal elastic band when they jumped over it-did not complete the task any faster, earning them lower scores. In other words, the fact that our subjects did not complete this task more quickly after the intervention could have been because they were paying more attention to performing the "jump-over" part of the sequence accurately. Indeed, the examiners observed that children's movements during this task were somewhat awkward at the time of the 1st test (T0) and pre-test (T1), but had become smoother and uninterrupted by the time of the post-test (T2). Thus, the slight (although non-significant) improvement on the jump-over- 
crawl-under task exhibited by our 4-year-old subjects could be attributed to their having learned how to perform the movements involved both more smoothly and accurately. Quantifying this sequence by observational assessments and motion analysis to investigate motor development remains a topic for future research.

Children's performance on the zigzag-run task was significantly improved after the intervention. Performance on this task is measured as the time required to run from a starting point to a distant point: children do not run straight ahead, instead zigzagging around obstacles. To perform well, runners need to tilt their body as they swiftly navigate the challenges, not keep their torso perfectly straight. Yagi, Wakita, and Mizutani (1989) found coordination ability to significantly correlate with sprinting ability and stride length ratio to height. Preschoolers have better coordination and better sprinting ability and longer strides. Some of the activities in our exercise program involve large movements through space in time with rhythms and bending the arm while stepping. It is plausible that regularly doing these activities in the intervention course-specifically, swiftly moving the legs in time with the music while compensating for instability in the arms-could have influenced children's sprinting ability and stride length, reducing completion time. Altogether, these findings suggest that regularly doing activities involving quick leg movements and simultaneous body tilt reduced our subjects' completion time on the zigzag-run, signifying improvements in coordination skills related to agility.

Children's performance on the side-stepping task was also significantly improved after the intervention. Hosokawa and Sato (2018) observed improvements in preschoolers' side-stepping performance after a "line training" intervention, which they attributed to practice and eventual acquisition of the movements required: specifically, skipping forward or backward along a line while alternately hopping to its left and right in different sequences. Thus, our subjects' improvements on the same task could have resulted from acquiring the motor patterns required through program activities involving changing direction when making step-related movements. Our subjects' step counts dramatically improved after our intervention, in both the 4- and 5-year-old groups, suggesting that active engagement in the program activities could have been responsible. Thus, it seems reasonable to conclude that our program's enthusiasm and engagement were responsible for the improvements in coordination skills, as measured by the side-stepping task.

Matsui et al. (1976) concluded that overall performance on a coordination test is influenced by training effects: specifically, children improve more when trained with drills and activities involving motor patterns similar to those assessed in the coordination test. Our rhythm-play exercise program includes activities involving jumping, but not the running movements of the zigzag run, nor any continuous jumping over and crawling under obstacles, as in the jump-over-crawl-under task. Likewise, our program's inclusion of activities involving jumping and children's repeated practice in addition to that is probably 
responsible for the improved coordination observed in the side-stepping task. Thus, a significant improvement on a coordination test after an exercise program intervention seems like a natural consequence when the two are similar in content, as was the case for the present study's side-stepping task. Nonetheless, repeated exposure to even dissimilar activities in an exercise program can enhance coordination skills and improve motor immaturity in preschool-age children. Iijima et al. (2010) have shown that repeated practice with various exercises involving physical movement (jumping up and down in particular) at a regular frequency and making adjustments to enliven the play environment are essential considerations that programs should implement to encourage improvements in motor skills, instead of training focused on learning proper techniques. Preschoolers gain experience with various exercises as they move their bodies in time with music in our exercise program. This program also includes activities where children shift their trajectory back-and-forth and left-to-right as they jump with one or both feet. Our program was not intended to drill a proper technique: instead, the vigorous activities in the program, which provided enjoyable exercise experiences, were most likely responsible for the observed improvements in their coordination skills.

In summary, our work offers several insights into how rhythmic-play exercise programs can influence coordination in preschool-age children. Our subjects showed improvements in coordination skills related to agility-as measured by the zigzag-run and side-stepping tasks-following completion of our program. Conversely, significant improvements in coordination skills related to dexterity-as measured by the jump-over-crawl-under task-were not observed; however, slight improvement signs were observed in the 4-year-old class. Our findings reveal that the exercise program we developed can influence coordination in preschool children, and confirm its effectiveness in acquiring these skills. Program activities had powerful effects on agility coordination skills, as measured by the zigzag-run and side-stepping tasks.

\section{Conclusion}

This study investigated the effects of an exercise program emphasizing rhythmic play on different aspects of motor coordination in preschool children. Specifically, the program's effectiveness was verified by comparing how well the same subjects performed different tasks in the CFT before and after a non-exercise intervention period and an exercise intervention period. Significant findings are summarized below:

1) Our program was not associated with significant improvements in jump-over-crawl-under performance. The five-year-old did not show significant improvement; however, the 4-year-old may have been influenced by the intervention, as evidenced by the slightly faster completion times after than before it (although not significantly so). This discrepancy suggests that young children should be given opportunities to acquire (fine-motor) coordination skills related 
to dexterity starting around the age of four.

2) Our program was associated with significant improvements in zigzag-run performance. Both 4- and 5-year-old children completed the task faster after than before the intervention, attributable to their movements becoming smoother and more refined due to the rhythmic activities in the program. Their success suggests that motor immaturity was improved through these activities, helping children learn how to deftly control their movements to swiftly perform the motor sequences involved in the exercise.

3) Our program was associated with significant improvements in side-stepping performance, suggesting that repeated experience with activities involving changing direction in the program, along with preschoolers' active engagement, helped to improve their coordination skills related to agility.

Our results suggest that one of the effects of exercise programs emphasizing rhythmic play for preschool-age children is their enhancement of coordination skills related to agility, as measured by the zigzag-run and side-stepping tasks. Conversely, our program did not yield significant improvements in coordination skills related to dexterity, as measured by the jump-over-crawl-under task; however, it could improve such skills in 4-year-olds. Efforts to help children acquire dexterity-related coordination should start around the age of four. Our results indicate that our exercise program had significant effects on coordination skills related to agility. Altogether, our findings confirm that the rhythmic-play exercise program examined here can effectively improve different aspects of coordination in preschool children. Their active engagement in its activities enhanced those effects. The difference between other exercise programs is that you are working on movement, such as jumping while aligning to the rhythm of the music. In addition, where there is no need to determine the good or bad of movement is a point worth noting.

Several outstanding issues should be addressed in future work. First, a control group should be established at a different kindergarten. Additionally, modified exercise programs targeting the improvement of coordination skills related to dexterity should be investigated, given our study population's lack of significant jump-over-crawl-under performance improvements. Finally, using motion analysis tools to clarify preschool children's coordination abilities would provide valuable information for answering questions of motor development in young children.

\section{Acknowledgements}

We would like to express our deepest gratitude to the preschool teachers and children who participated in this study.

\section{Conflicts of Interest}

The authors declare no conflicts of interest regarding the publication of this paper. 


\section{References}

Evridiki, Z., Aggeliki, T., \& Vassiliki, D. (2004). The Effects of a Developmentally Appropriate Music and Movement Program on Motor Performance. Early Childhood Research Quarterly, 19, 631-642. https://doi.org/10.1016/j.ecresq.2004.10.005

Furuichi, H. (1995). A Consideration of Many Sides in Body Expression Activities in Children. Educare, 16, 21.

Hirai, H., \& Kasahara, A. (2016). Study of the Method to Adopt Coordination-training for Physical Education Classes in Elementary School, and Improve Physical Strength, Athletic Capability Measurements: Practice in Inabe City, Mie. Practical Education Research (Chubu Gakuin Universityl Junior College), 1, 181-188.

Hosokawa, K., \& Sato, T. (2018). Line Training for Young Children Improves Coordination. Research Journal of Sports Performance, 10, 252-269.

Iijima, H., Kizuka, T., Hayami, T., Iwai, M., Itaya, A., \& Suzuki, H. (2010). Effect of Exercise Programs on an Unstable Support Surface for Moter Ability of Infants. Japan Journal of Human Growth and Developmental Research, 2010, 10-20. https://doi.org/10.5332/hatsuhatsu.2010.47 10

Ikai, M. (1967). Coordination: Physiological Considerations. Japan Journal of Physical Education, Health and Sport Sciences, 11, 5-10.

Ishikawa, T., Takada, N., Ono, M., Katsube, A., Matsuura, Y., Miyamaru, M. et al. (1987). Summary of Research Findings on Coordination. Journal of Health, Physical Education and Recreation, 15, 75-87.

Kamimaru, K. (2010). Conditioning Exercises as Coordination Training. International Human Studies (Kagoshima Immaculate Heart University), 17, 45-57.

Katoh, K., \& Komori, S. (2020). A Study on the Order of Acquisition of Jump Movements in Young Children Based on Observation and Evaluation. Japan Journal of Human Growth and Development Research, 2020, 32-43. https://doi.org/10.5332/hatsuhatsu.2020.86 32

Kozuka, K., Kuga, A., \& Watanabe, T. (2010). Implementation of Coordination Training Targeted for Preschool Children. A Case Study at MizugidaiDaycare in Hanno City. Surugadai University Rongo, 41, 131-144.

Kurimoto, E., Asami, T., Shibukawa, K., Matsuura, Y., \& Katsube, A. (1981). Physical Education Center Coordination Ability Field Test Final Format: Coordination Ability Test Review Committee Report. Journal of Health, Physical Education and Recreation, 9, 207-212.

Maehashi, A. (2017). Compass - Physical Education in Early Childhood: Cultivating the Mind through Movement (p. 10). Tokyo: Kenpakusha.

Matsui, S., Katsube, A., Mizutani, S., \& Wakita, H. (1976). A Study on Practiced Effect for Increasing Ability of Coordination and Integration. Journal of Health, Physical Education and Recreation, 4, 158-169.

Matsuura, Y. (1998).On The Coordination Ability. Report of Research Center for Physical Education Fitness Improvement Science 1998. Journal of Health, Physical Education and Recreation, 27, 137-146.

MEXT (1969). National Curriculum Standards for Elementary Schools: Physical Education (pp. 2-12). Tokyo: Toyokan Publishing.

MEXT (2017). National Curriculum Standards for Kindergartens. https://www.mext.go.jp/a menu/shotou/new-cs/youryou/you/index.htm

Ministry of Education, Culture, Sports, Science and Technology (MEXT) (1968). National 
Curriculum Standards for Elementary Schools: Physical Education. https://erid.nier.go.jp/files/COFS/s43e/chap2-8.htm

Ministry of Health, Labour and Welfare (2010). 2010 National Growth Survey on Preschool Children. https://www.mhlw.go.jp/stf/houdou/0000042861.html

Nakamura, K., Takenaga, R., Masahiro, K., Kawazoe, K., Shinohara, T., Yamamoto, T. et al. (2011). Development of Fundamental Motor Pattern Using the Observational Evaluation Method in Young Children. Japan Journal of Human Growth and Development Research, 2011, 1-18. https://doi.org/10.5332/hatsuhatsu.2011.51 1

Research Center in Physical Education (RCPE)-Expert Committee on Coordination (1981). Final Format of RCPE Coordination Field Test-A Coordination Test Review Committee Report. Report of Research Center in Physical Education, 9, 207-212.

Science Council of Japan (SCJ)—Committee on Health and Human Life Science-Subcommittee on Health and Sports Science (2017). Proposal: Striving for Healthy Development of Movements in Children: Basic Movements Are Dangerous. http://www.scj.go.jp/ja/info/kohyo/pdf/kohyo-23-t245-1.pdf

Takahara, K., Sunami, Y., \& Taki, N. (2015). The Relationship between Play Activities and Motor Abilities among Young Children. Fukuoka Jogakuin University Bulletin: Human Relations, 16, 87-97.

Takenawa, M., Takeda, T., Kikuchi, H., \& Nakagawa, K. (2008). Teaching Program for Kids in Aerobics. Bulletin of the Continuing Education Research Institute (Hokkaido AsaiGakuen University), 11, 213-227.

Tanaka, C., Hikihara, Y., Sasaki, R., Ando, T., Naka, T., Usui, C., \& Tanaka, S. (2017). Relationship between the Qualitative Evaluation of Fundamental Motor Skills and Quantitative Motor Performance in Preschool Children. Japan Journal of Human Growth and Development Research, 76, 8-15.

https://doi.org/10.5332/hatsuhatsu.2017.76 8

Tomita, H., Otobuchi, R., \& Matsushima, A. (2014). The Effect of an Exercise Program That Includes Rhythmic Motion on the Sense of Rhythm and Physical Fitness of Young Children. Bulletin of the Shizuoka Institute of Science and Technology, 22, 51-56.

Ueta, K., Watahiki, K., Ishibashi, K., Sakamoto, H., Morito, T., \& Umino, K. (2006). An Exploration of the Physical Education Class, Which Incorporates Coordination Training: Incorporation into "KARADA TSUKURI UNDO". Research Bulletin of Naruto University of Education, 21, 370-377.

Umezaki, S., Nakatani, T., Yamamoto, D., Nakasuka, T., \& Hashimoto, M. (2013). Effects of Coordination Exercise on the Motion Capacity of Preschool Children: Quantitative and Qualitative Changes in Throwing and Catching Ability. Japan Journal of Human Growth and Developmental Research, 59, 27-40.

https://doi.org/10.5332/hatsuhatsu.2013.27

Yagi, N., Wakita, H., \& Mizutani, S. (1989). Running Ability Observed from Muscular Power and Coordination for Preschool Children. Bulletin of the Faculty of Education Mie University (Natural Science), 40, 81-91.

Yanagisawa, A. (1994). Research on Physical Exercise to Improve Coordination: Examination of the Effect of Systematic Instruction of Jump Box/Mat Exercise on the Adjustment Ability of Preschool Children. Bulletin of Matsumoto Junior College Research, 4, 81-93.

Yasumitsu, T., \& Nogawa, H. (2010). Effectiveness of a Coordination Exercise Program during School Recess of Elementary School: Focusing on Agility. Research Journal of Sports Performance, 2, 233-245. 
Yoshida, I., \& Iwasaki, H. (2014). Physical-Motor-Play Instruction in the Kindergarten and Cognition of Kindergarten Teacher to Physical-Motor-Play Instruction: Study of Satisfaction for the Physical-Motor-Play Instruction in Kindergarten and Technical teaching Orientation That the Kindergarten Teachers Have. Japan Journal of Human Growth and Developmental Research, 2014, 18-24.

https://doi.org/10.5332/hatsuhatsu.2014.64 18

Yoshimi, E. (2020a). Study on the Cooperative Movement of Infants: Focus on the Richness of Movement Seen in Rhythmic Movements (2). Annual Reports of Studies. Tokiwakai Junior College, 48, 87-102.

Yoshimi, E. (2020b). Study on the Cooperative Movement of Infants: Focus on the Richness of Movement Seen in Rhythmic Movements (3). Tokiwakai Junior College Early-Childhood Education Journal, 36, 30-43.

Yoshimi, E., \& Katayama, H. (2019). Study on the Cooperative Movement of Infants: Focus on the Richness of Movement Seen in Rhythmic Movements (1). Tokiwakai Junior College Early-Childhood Education Journal, 35, 58-71.

Yoshimi, E., Nomura, T., \& Kida, N. (2021). A Study of Young Children's Coordinated Movement-The Effects of a Rhythmic-Play Exercise Program on Physical-Expression Ability. Advances in Physical Education, 11, 118-134.

https://doi.org/10.4236/ape.2021.111009 